\title{
Resection of a Nonampullary Adenoma with the New Gastroduodenal Full-Thickness Resection Device
}

\author{
Maximilian Gericke ${ }^{1}$ Matthias Mende ${ }^{1}$ Dirk Hartmann ${ }^{2} \quad$ Siegbert Faiss ${ }^{1}$ \\ ${ }^{1}$ Department of Gastroenterology, Sana Klinikum Lichtenberg, \\ Berlin, Germany \\ 2Department of Gastroenterology, Katholisches Klinikum Mainz, \\ Germany

\begin{abstract}
Address for correspondence Dr. med. Maximilian Gericke, Sana Klinikum Lichtenberg, Klinik für Innere Medizin I Schwerpunkt, Gastroenterologie, Fanningerstraße 32,10365 Berlin, Germany (e-mail: maximilian.gericke@sana-kl.de).
\end{abstract}

J Digest Endosc:2020;11:242-244

\section{Introduction}

Adenomas in the duodenum can develop spontaneously or have an association to hereditary syndromes. They can develop into malignant adenocarcinomas, following the adenoma-carcinoma sequence, shown in colorectal adenomas. ${ }^{1}$ About 5\% of duodenal adenomas progress into carcinomas, therefore a resection should be performed. ${ }^{1}$ Removal of subepithelial tumors in the duodenum should be done if there are symptoms (e.g., bleeding) or suspicion of malignancy. Surgical resection has a higher morbidity versus endoscopic resection. ${ }^{2}$ Nevertheless, endoscopic resection of those lesions has a significant risk of complications such as perforation or bleeding. ${ }^{3,4}$

Endoscopic full-thickness resection (EFTR) using the full-thickness resection device (FTRD, Ovesco Endoscopy AG, Tuebingen, Germany) has been reported to be effective and safe in the colorectum. ${ }^{5,6}$ Technical success of EFTR in the duodenum has been shown in previous studies. ${ }^{7,8}$ The new gastroduodenal FTRD (Ovesco Endoscopy AG, Tuebingen, Germany) was recently Communauté Européenne (CE) for EFTR of duodenal lesions. Compared with the colonic FTRD the diameter is $19.5 \mathrm{~mm}$ and has an integrated guide wire and balloon for dilatation. We now present the case of a resection of a recurrent nonlifting duodenal adenoma using the new gastroduodenal FTRD.

\section{Case Report}

A 37-year-old white man was admitted to our hospital for endoscopic removal of a recrudescent adenoma. The adenoma in the flexura duodeni inferior had a central scarred retraction and biopsy-proven low-grade intraepithelial neoplasia (IEN). An endoscopic mucosal resection (EMR) was performed 6 and 4 months before presentation at our institution. The patient did not have any symptoms. Medical history contained hypertension and an episode of depression. Physical examination and laboratory workup did not show any pathology. The patient gave written informed consent.

Before resection, the margins of the $10-\mathrm{mm}$ adenoma were marked with argon-plasma coagulation (APC) with a standard gastroscope. Then we switched to the gastroduodenal FTRD. After balloon dilatation of the upper esophageal sphincter (UES) of $20 \mathrm{~mm}$, a passage with the FTRD system was not possible. No relevant mucosal damage was detected. The patient was discharged without pain or bleeding signs. 
A new attempt was done 2 months later. After boogying on 17 to $19 \mathrm{~mm}$ with bougies, the endoscope with FTRD cap was used. After balloon dilatation of the upper sphincter of the esophagus of $20 \mathrm{~mm}$, a passage with the FTRD system was again not possible. The pressure was increased and after switching the patient into supine position, the passage was eventually successful. The endoscope was then advanced into the duodenum. The grasping forceps were advanced through the working channel. The lesion was pulled into the cap to incorporate a double, full-thickness layer of the duodenal wall. The over-the-scope clip (OTSC) was then deployed, and the tissue above the clip was immediately resected with the snare ( $\boldsymbol{-}$ Figs. $\mathbf{1}$ and $\mathbf{2}$ ). The resected adenoma was retrieved. There were no signs for bleeding or perforation. A control gastroscopy one day after showed a correctly placed OTSC. A minor contact bleeding was treated by APC. The histological workup of the specimen $(28 \mathrm{~mm} \times 22 \mathrm{~mm})$ showed a 3-mm tubular adenoma with low-grade IEN and complete (R0) resection. The patient was discharged after 2 days without any further complications.

\section{Follow-up}

A gastroscopy 10 weeks after the EFTR showed no recurrence of the adenoma. The OTSC was still in situ. There were no adverse events.

\section{Discussion}

Compared with the colon, the duodenum has a special anatomy making endoscopic resection of adenomas or subepithelial tumors more difficult. The duodenal wall is thinner than in the colon, the risk of bleeding is higher, and it is less flexible due to its fixation in the retroperitoneum. However, endoscopic resection of lesions has a lower morbidity than the surgical approach. ${ }^{2}$
EMR is the standard procedure for resection of duodenal nonampullary adenomas. Success rates for complete removal is high. ${ }^{2}$ The risk of periprocedural bleeding is up to $25 \%$, with delayed bleeding up to $12 \%{ }^{3,9}$ Moreover, the risk of perforation is up to $5 \%{ }^{9}$ In comparison with endoscopic submucosal dissection (ESD) in the duodenum, the risk of perforation is up to $35 \%$, for this reason duodenal ESD is not recommended. ${ }^{10}$ EFTR is another reasonable technique for resection of nonlifting adenomas or submucosal tumors. The FTRD in the colorectum has proven its efficacy with acceptable complication rates. ${ }^{6,8}$

FTRD in the duodenum has shown promising results in two previous studies including 24 patients in total with "difficult" adenomas (such as adenomas with nonlifting sign) or subepithelial tumors.,8 Minor bleeding occurred in approximately $20 \%$ of patients, no major bleeding or perforation was detected. Complete resection (R0) was achieved in $69.5 \%$.

We report about a case of successful resection of a duodenal adenoma using the new gastroduodenal FTRD (Ovesco, Tuebingen) which was recently CE-labeled. Its outer diameter is $19.5 \mathrm{~mm}$ that is $1.5 \mathrm{~mm}$ thinner than the colonic FTRD. A limitation to FTRD in the upper gastrointestinal tract is the need for balloon dilation of the UES and pylorus to allow passage of the device. The passage of UES and pylorus is also the most challenging part when using the gastroduodenal FTRD in the upper gastrointestinal tract. Therefore, a guide wire and balloon for dilatation are integrated. Despite the lower diameter, passage might not be possible as we described in our case. Shifting to supine position and using a higher pressure for dilatation may enable passage, although the risk of injury or perforation seems to be higher. An elective removal of the OTSC to our experience is not necessary. However, if complications occur, such as luminal obstruction, clipping of extraluminal tissues, or ulceration of the surrounding area or patients' discomfort, the OTSC should be removed.

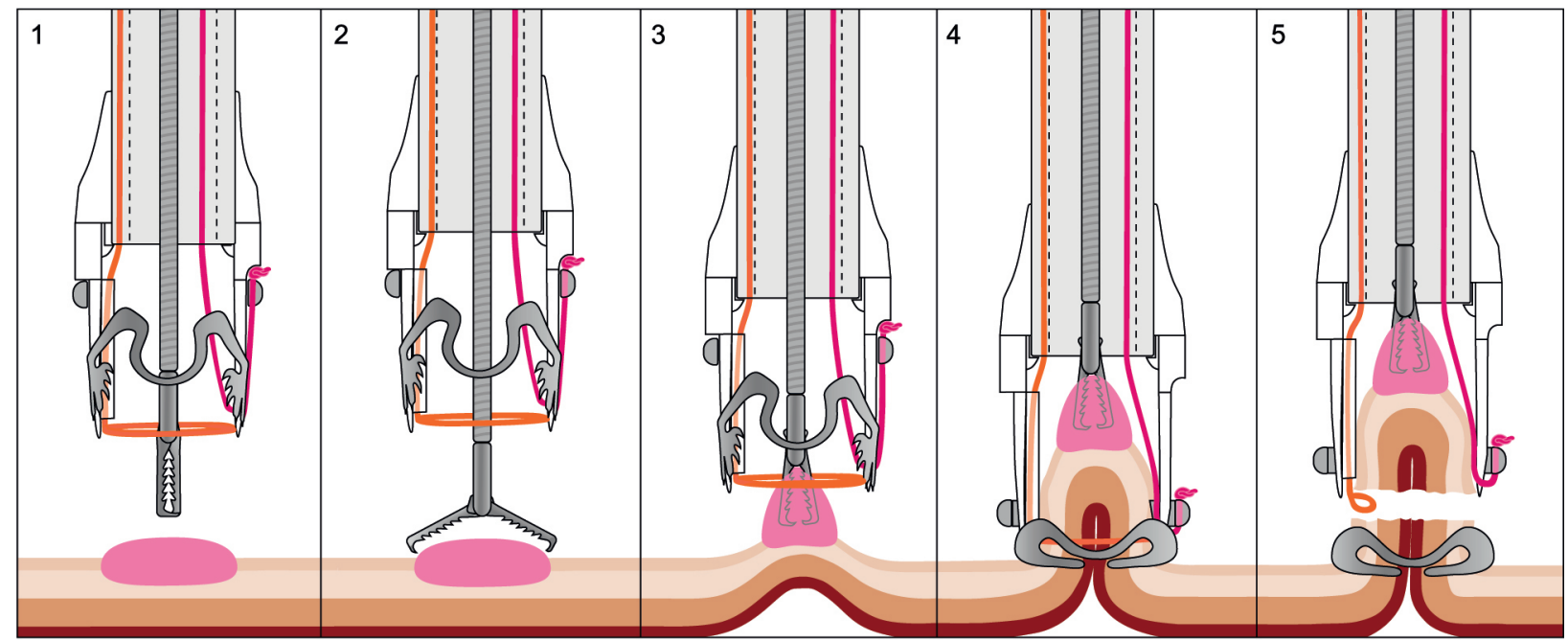

Fig. 1 Illustration of the FTRD procedure. (1 and 2) Grasping forceps are advanced through the working channel of the endoscope. (3) The target lesion is grasped and pulled into the cap. (4) The OTSC is deployed and creates a full-thickness plication of the gastrointestinal wall. (5) The pseudopolyp is resected above the OTSC with the preloaded snare (Courtesy of Ovesco Endoscopy AG, Tuebingen, Germany, with permission). FTRD, full-thickness resection device; OTSC, over-the-scope clip. 

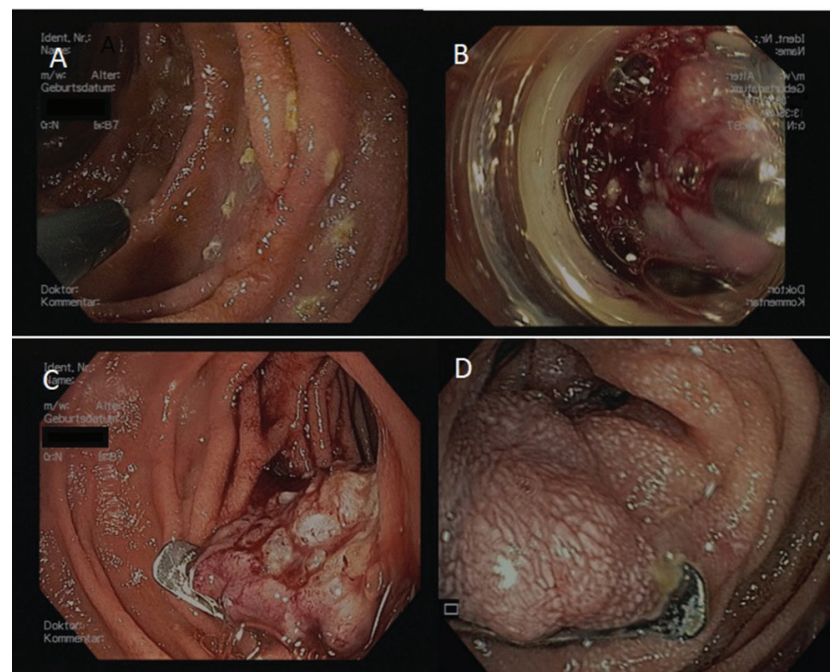

Fig. 2 Full-thickness resection of an adenoma in the duodenum: (A) marks around the adenoma; (B) grasping the adenoma; (C) correctly placed OTSC; (D) control after 3 months with OTSC in situ and cicatricial tissue. OTSC, over-the-scope clip.

Due to a limited number of patients treated with FTRD in the duodenum, the incidence of complications cannot be evaluated properly. Therefore, more studies are needed.

The size of the lesions should not exceed $25 \mathrm{~mm}$ because the risk of nonsuccessful resection rises with the lesion size. ${ }^{8}$ That limits the use of FTRD in larger flat adenomas extending over several folds. In comparison to EFTR in the colorectum, lesions up to $30 \mathrm{~mm}$ could be resected with sufficient success. ${ }^{6}$ It is not possible to resect tumors in the ampullary duodenum via FTRD due to the risk of clipping the common bile duct. We strongly recommend localizing the papilla before EFTR and to make sure that there is a distance of at least $20 \mathrm{~mm}$ to the papilla.

\section{Conclusion}

In conclusion, the gastroduodenal FTRD in the duodenum is a promising new device which makes it possible to resect lesions not suiting for EMR and thus to avoid surgery.
Randomized-controlled trials are needed to evaluate the safety and success of the gastroduodenal FTRD.

\section{Conflict of Interests}

The authors declare there are no conflicts of interest.

\section{References}

1 Okada K, Fujisaki J, Kasuga A, et al. Sporadic nonampullary duodenal adenoma in the natural history of duodenal cancer: a study of follow-up surveillance. Am J Gastroenterol 2011;106(2):357-364

2 Gaspar JP, Stelow EB, Wang AY. Approach to the endoscopic resection of duodenal lesions. World J Gastroenterol 2016;22(2):600-617

3 Aschmoneit-Messer I, Richl J, Pohl J, Ell C, May A. Prospective study of acute complication rates and associated risk factors in endoscopic therapy for duodenal adenomas. Surg Endosc 2015;29(7):1823-1830

4 Lim CH, Cho YS. Nonampullary duodenal adenoma: current understanding of its diagnosis, pathogenesis, and clinical management. World J Gastroenterol 2016;22(2):853-861

5 Valli PV, Mertens J, Bauerfeind P. Safe and successful resection of difficult GI lesions using a novel single-step full-thickness resection device (FTRD) Surg Endosc 2018;32(1):289-299

6 Schmidt A, Beyna T, Schumacher B, et al. Colonoscopic full-thickness resection using an over-the-scope device: a prospective multicentre study in various indications. Gut 2018;67(7):1280-1289

7 Schmidt A, Meier B, Cahyadi O, Caca K. Duodenal endoscopic full-thickness resection (with video) Gastrointest Endosc 2015;82(4):728-733

8 Bauder M, Schmidt A, Caca K. Endoscopic full-thickness resection of duodenal lesions-a retrospective analysis of 20 FTRD cases. United European Gastroenterol J 2018;6(7):1015-1021

9 Yamasaki Y, Uedo N, Takeuchi Y, Ishihara R, Okada H, Iishi H. Current status of endoscopic resection for superficial nonampullary duodenal epithelial tumors. Digestion 2018;97(1):45-51

10 Yamamoto H, Miura Y. Duodenal ESD: conquering difficulties. Gastrointest Endosc Clin N Am 2014;24(2):235-244 